RUB-TPII-18/96

hep-ph/9709221

\title{
Semileptonic decay constants of octet baryons in the chiral quark-soliton model
}

\author{
Hyun-Chul Kim, Maxim V. Polyakov 1 , Michał Praszałowicz \\ Institute for Theoretical Physics II, \\ Ruhr-University Bochum, \\ D-44780 Bochum, Germany
}

(August, 1997)

\begin{abstract}
Based on the recent study of the magnetic moments and axial constants within the framework of the chiral quark-soliton model, we investigate the baryon semileptonic decay constants $\left(f_{1}, f_{2}\right)$ and $\left(g_{1}, g_{2}\right)$. Employing the relations between the diagonal transition matrix elements and off-diagonal ones in the vector and axial-vector channels, we obtain the ratios of baryon semileptonic decay constants $f_{2} / f_{1}$ and $g_{1} / f_{1}$. The $F / D$ ratio is also discussed and found that the value predicted by the present model naturally lies between that of the Skyrme model and that of the nonrelativistic quark model. The singlet axial constant $g_{A}^{(0)}$ can be expressed in terms of the $F / D$ ratio and $g_{A}^{(3)}$ in the present model and turns out to be small. The results are compared with available experimental data and found to be in good agreement with them. In addition, the induced pseudotensor coupling constants $g_{2} / f_{1}$ are calculated, the $\mathrm{SU}(3)$ symmetry breaking being considered. The results indicate that the effect of SU(3) symmetry breaking might play an important role for some decay modes in hyperon semileptonic decay.
\end{abstract}

PACS: 12.39.Fe, 13.30.Ce, 14.20.Jn

Typeset using REVTEX

\footnotetext{
*Dedicated to Professor Richard Lemmer on the occasion of his 65th birthday.

${ }^{\dagger}$ On leave of absence from PNPI, Gatchina, St.Petersburg 188350, Russia

$\ddagger$ On leave of absence from Institute of Physics, Jagellonian University, Cracow, Poland
} 


\section{INTRODUCTION}

Baryon semileptonic decays have played an important role in various facets to understand the structure of baryons. For example, they provide information on the Cabibbo-KobayashiMaskawa (CKM) angles $\left|V_{u d}\right|$ and $\left|V_{u s}\right|$ as well as the $F / D$ ratio. Recently, baryon semileptonic decays have gained new interest in respect of a series of experiments measuring the first moment of the spin-dependent structure function $g_{1}(x)$ [1,2, 3, 4], since it is related to $\mathrm{SU}(3)$ invariant matrix elements of hyperon semileptonic processes $F$ and $D$.

A recent high-precision measurement of $\Sigma^{-} \rightarrow n+e^{-}+\bar{\nu}$ [5] shows a hint that the effect of $\mathrm{SU}(3)$ symmetry breaking might be important to describe baryon semileptonic decays. Another experiment measuring $g_{1} / f_{1}$ in $\Lambda \rightarrow p+e^{-}+\bar{\nu}$ was conducted by J. Dworkin et al. with high statistics [6]. The result of Ref. [6] prefers the hypothesis that the weak magnetism is less than the CVC prediction $\left(f_{2} / f_{1}=0.97\right)$. In fact, Ref. [6] obtained $f_{2} / f_{1}=0.15 \pm 0.30$ at which the fit yields the minimum of $\chi^{2}$. This value is really far from the CVC hypothesis. Also from the theoretical point of view there were already serious doubts about the strong postulate of exact $\mathrm{SU}(3)$ symmetry in the Cabibbo theory [7,8,9].

The effects of $\mathrm{SU}(3)$ symmetry breaking in baryon semileptonic decays have been extensively studied from various points of view [9,10,11, 12,13. Donoghue et al. [9] made a careful analysis of hyperon semileptonic decays, considering the pattern of symmetry breaking based on the quark model. It was asserted in Ref. [9] that SU(3) breaking comes from two sources: the mismatch of the wave functions for the quarks and the recoil effect. However, Roos reanalyzed hyperon semileptonic decays [10] including recent data and showed that the scheme of symmetry breaking by Donoghue et al. fails to fit correctly the $g_{1} / f_{1}$ ratio for $\Lambda \rightarrow p+e^{-}+\bar{\nu}$. The mismatch of strange-quark wave functions worsens the fit. Only by introducing a substantial second-class axial coupling $g_{2}$ in the $\Lambda \rightarrow p+e^{-}+\bar{\nu}$, one could fit the data [10]. Avenarius [11] studied also SU(3) symmetry breaking in semileptonic hyperon decays, based on the Ansatz that SU(3) symmetry in the polarization at the current quark level is kept while $\mathrm{SU}(3)$ symmetry at the constituent quark level is broken. The result was that with $\mathrm{SU}(3)$ symmetry broken the $F / D$ ratio $(0.73 \pm 0.09)$ turned out to be larger than that of the case of SU(3) symmetry $(0.59 \pm 0.02)$. Ehrnsperger and Schäfer [12] came to a rather different conclusion. They showed that the effects of $\mathrm{SU}(3)$ symmetry breaking lead to a reduction of the $F / D$ ratio $(0.49 \pm 0.08)$. Quite recently, Ratcliffe [13 reexamined $\mathrm{SU}(3)$ symmetry breaking effects in hyperon semileptonic decays. What he obtained is that $F / D=0.582$ for an $\mathrm{SU}(3)$ symmetric fit and $F / D=0.570$ for an $\mathrm{SU}(3)$ breaking fit. The result of Ref. [13] indicates that the effects of SU(3) symmetry breaking is rather tiny. There have been also similar discussions related to the validity of $\mathrm{SU}(3)$ symmetry in the context of the spin structure of the proton. In particular, Lipkin strongly criticized the use of SU(3) symmetry in studying the spin structure of the proton [14]. The topic of SU(3) symmetry breaking in hyperon semileptonic decays seems to be evidently far from the settlement yet and very difficult to be analyzed without relying on particular Ansätze.

Recently, we investigated the magnetic moments of the baryon octet [15] and the axial constants of the nucleon [16] within the framework of the chiral quark-soliton model ( $\chi$ QSM) [17], taking into account the $1 / N_{c}$ rotational corrections and linear $m_{s}$ corrections which furnish the effect of $\mathrm{SU}(3)$ symmetry breaking. The magnetic moments and axial constants which are given in terms of diagonal matrix elements can be, however, related to 
the off-diagonal transition form factors, i.e. semileptonic weak form factors $f_{1}, f_{2}$ and $g_{1}$. Therefore, we can easily evaluate them, using former calculations. The presence of the $m_{s}$ corrections allows the nonvanishing values of induced pseudotensor coupling constants $g_{2}$.

The large $N_{c}$ limit $\left(N_{c} \rightarrow \infty\right)$ provides a useful guideline in understanding the low-energy properties of the baryon systematically [18], though in reality $N_{c}$ is equal to 3 . In the large $N_{c}$, the nucleon can be viewed as a classical soliton of the pion field. An example of the dynamical realization of this idea is given by the Skyrme model [19]. However, the $\chi$ QSM presents a more realistic picture than the Skyrme model. In the light of chiral perturbation theory, the effective chiral action on which the $\chi$ QSM is based contains automatically the four-derivative Gasser-Leutwyler terms [20] and the venerable Wess-Zumino term [21] with correct coefficients [22,23,24]. Moreover, the $\chi$ QSM interpolates between the Skyrme model and the nonrelativistic model (NRQM) [25,26], because the $\chi$ QSM is ideologically close to the Skyrme model in the limit of large soliton size while as the size of the soliton approaches zero the $\chi$ QSM reproduces the results of the NRQM.

The aim of the present paper is to investigate the semileptonic decay constants $\left(f_{1}, f_{2}\right)$ and $\left(g_{1}, g_{2}\right)$ within the framework of the $\chi$ QSM.

The outline of the paper is as follows: In the next section, we sketch briefly the basic formalism of the $\chi$ QSM. In section III, we first discuss the semileptonic decay constants in $\mathrm{SU}(3)$ flavor symmetry. As it should be, it is shown that the induced pseudotensor coupling constant $g_{2}(0)$ vanishes in $\mathrm{SU}(3)$ symmetry within the framework of the $\chi \mathrm{QSM}$. The $F / D$ ratio is also discussed. The singlet axial constant $g_{A}^{(0)}$ is shown to be related to the $F / D$

ratio and the axial constant $g_{A}^{(3)}$. Considering the strange quark mass $m_{s}$, we discuss the effect of $\mathrm{SU}(3)$ symmetry breaking on the coupling constants $f_{1}, f_{2}$ and $g_{1}$. The $g_{2}$ is also evaluated. We compare the results with those obtained in the case of SU(3) symmetry. The deviations from the Cabibbo theory are discussed in detail. In section IV, we summarize the present work and draw the conclusion.

\section{GENERAL FORMALISM}

The transition matrix element $\mathcal{M}_{B_{1} \rightarrow B_{2} l \bar{\nu}_{l}}$ for the process $B_{1} \rightarrow B_{2} l \bar{\nu}_{l}$ can be written as

$$
\mathcal{M}_{B_{1} \rightarrow B_{2} l \bar{\nu}_{l}}=\frac{G}{\sqrt{2}}\left(\begin{array}{c}
V_{u d}(\text { for } \Delta S=0) \\
V_{u s}(\text { for }|\Delta S|=1)
\end{array}\right)\left\langle B_{2}\left|J_{\mu}^{W}\right| B_{1}\right\rangle \bar{u}_{l}\left(p_{l}\right) \gamma^{\mu}\left(1-\gamma_{5}\right) u_{\bar{\nu}_{l}}\left(p_{\nu}\right),
$$

where $G$ denotes the effective Fermi coupling constant and $V_{u d}, V_{u s}$ stand for the CabibboKobayashi-Maskawa angles. The leptonic current $\bar{u}_{l}\left(p_{l}\right) \gamma^{\mu}\left(1-\gamma_{5}\right) u_{\bar{\nu}_{l}}\left(p_{\nu}\right)$ is the known part. The hadronic weak current $J_{\mu}^{W}$ has following spin and flavor structures:

$$
J_{\mu}^{W}=\left\{\begin{array}{c}
\bar{\psi}(x) \gamma_{\mu}\left(1-\gamma_{5}\right) \frac{1}{2}\left(\lambda_{1} \pm i \lambda_{2}\right) \psi(x), \text { for } \Delta S=0 \\
\bar{\psi}(x) \gamma_{\mu}\left(1-\gamma_{5}\right) \frac{1}{2}\left(\lambda_{4} \pm i \lambda_{5}\right) \psi(x), \text { for }|\Delta S|=1
\end{array}\right.
$$

The transition matrix element of the hadronic weak current $\left\langle B_{2}\left|J_{\mu}^{W}\right| B_{1}\right\rangle$ can be expressed in terms of six independent form factors:

$$
\left\langle B_{2}\left|J_{\mu}^{W}\right| B_{1}\right\rangle=\bar{u}_{B_{2}}\left(p_{2}\right)\left[\left\{f_{1}\left(q^{2}\right) \gamma_{\mu}-\frac{i f_{2}\left(q^{2}\right)}{M_{1}} \sigma_{\mu \nu} q^{\nu}+\frac{f_{3}\left(q^{2}\right)}{M_{1}} q_{\mu}\right\}\right.
$$




$$
\left.-\left\{g_{1}\left(q^{2}\right) \gamma_{\mu}-\frac{i g_{2}\left(q^{2}\right)}{M_{1}} \sigma_{\mu \nu} q^{\nu}+\frac{g_{3}\left(q^{2}\right)}{M_{1}} q_{\mu}\right\} \gamma_{5}\right] u_{B_{1}}\left(p_{1}\right)
$$

with the momentum transfer $q=p_{2}-p_{1}$. The form factors $f_{i}$ and $g_{i}$ are real quantities depending only on the square of the momentum transfer in the case of $C P$-invariant processes. We can safely neglect $f_{3}$ and $g_{3}$ for the reason that on account of $q_{\mu}$ their contribution to the decay rate is proportional to the ratio $\frac{m_{l}^{2}}{M_{1}^{2}} \ll 1$, where $m_{l}$ represents the mass of the lepton $\left(e\right.$ or $\mu$ ) in the final state and $M_{1}$ that of the baryon in the initial state .

It is already well known how to deal with the hadronic matrix elements such as $\left\langle B_{2}\left|J_{\mu}^{W}\right| B_{1}\right\rangle$ in the $\chi$ QSM (for a review see [27]). Hence, we shall briefly explain how to calculate them with regard to the semileptonic processes . The $\chi \mathrm{QSM}$ is characterized by a low-momenta QCD partition function [28] which is given by a functional integral over 8 pseudoscalar and quark fields:

$$
\mathcal{Z}=\int \mathcal{D} \psi \mathcal{D} \psi^{\dagger} \mathcal{D} \pi^{a} \exp \left(\int d^{4} x \psi^{\dagger} \beta\left(-i \not \partial+\hat{m}+M U^{\gamma_{5}}\right) \psi\right)
$$

Integrating out quark fields leave us with the effective chiral action

$$
\mathcal{Z}=\int \mathcal{D} \pi^{a} \exp \left(-S_{\text {eff }}[\pi]\right)
$$

where

$$
S_{\text {eff }}=-\operatorname{Sp} \ln \beta\left(-i \not \partial+\hat{m}+M U^{\gamma_{5}}\right)
$$

with the pseudoscalar chiral field

$$
U^{\gamma_{5}}=\exp \left(i \pi^{a} \lambda^{a} \gamma_{5}\right)=\frac{1+\gamma_{5}}{2} U+\frac{1-\gamma_{5}}{2} U^{\dagger}
$$

$\hat{m}$ is the matrix of the current quark mass given by

$$
\hat{m}=\operatorname{diag}\left(m_{u}, m_{d}, m_{s}\right)=m_{0} \mathbf{1}+m_{8} \lambda_{8}
$$

$\lambda^{a}$ represent the usual Gell-Mann matrices normalized as $\operatorname{tr}\left(\lambda^{a} \lambda^{b}\right)=2 \delta^{a b}$. Here, we have assumed isospin symmetry $\left(m_{u}=m_{d}=\bar{m}\right)$. $M$ stands for the dynamical quark mass arising from the spontaneous chiral symmetry breaking, which is in general momentumdependent [28]. We regard $M$ as a constant and employ the proper-time regularization for convenience. The $m_{0}$ and $m_{8}$ in Eq. (8) are respectively defined by

$$
m_{0}=\frac{2 \bar{m}+m_{s}}{3} \simeq \frac{m_{s}}{3}, \quad m_{8}=\frac{\bar{m}-m_{s}}{\sqrt{3}} \simeq \frac{-m_{s}}{\sqrt{3}}
$$

The operator $i D$ is expressed in Euclidean space in terms of the Euclidean time derivative $\partial_{\tau}$ and the Dirac one-particle Hamiltonian $H\left(U^{\gamma_{5}}\right)$

$$
i D=\partial_{\tau}+H\left(U^{\gamma_{5}}\right)+\beta \hat{m}
$$

with 


$$
H\left(U^{\gamma_{5}}\right)=\frac{\vec{\alpha} \cdot \nabla}{i}+\beta M U^{\gamma_{5}}
$$

$\beta$ and $\vec{\alpha}$ are the well-known Dirac Hermitian matrices. The $U$ is assumed to have a structure corresponding to the embedding of the $\mathrm{SU}(2)$-hedgehog into $\mathrm{SU}(3)$ :

$$
U_{c}=\left(\begin{array}{cc}
U_{0} & 0 \\
0 & 1
\end{array}\right)
$$

with

$$
U_{0}=\exp (i \hat{r} \cdot \vec{\tau} P(r))
$$

$P(r)$ is called profile function. The partition function of Eq.(任 can be simplified by the saddle point approximation which is exact in the large $N_{c}$ limit. One ends up with a stationary profile function $P(r)$ which is evaluated by solving the Euler-Lagrange equation corresponding to $\delta S_{\text {eff }} / \delta P(r)=0$. This gives a static classical field $U_{0}$. The soliton is quantized by introducing collective coordinates corresponding to $\mathrm{SU}(3)_{\mathrm{f}}$ rotations of the soliton in flavor space (and simultaneously $\mathrm{SU}(2)_{\text {spin }}$ in spin space)

$$
U(t, \vec{x})=R(t) U_{c}(\vec{x}) R^{\dagger}(t),
$$

where $R(t)$ is a time-dependent $\mathrm{SU}(3)$ matrix. The quantum states from this quantization are identified with the $\mathrm{SU}(3)$ baryons according to their quantum numbers. In the large $N_{c}$ limit, the angular velocity of the soliton $\Omega=R^{\dagger}(t) \dot{R}(t)$ can be regarded as a small parameter, so that we can use it as an expansion parameter. After the rotation, the Dirac differential operator Eq.(10) can be expressed as

$$
i \tilde{D}=\left[\partial_{\tau}+H\left(U^{\gamma_{5}}\right)+\Omega(t)+\gamma_{4} R^{\dagger}(t) \hat{m} R(t)\right] .
$$

Then the propagator $(i \tilde{D})^{-1}$ can be expanded with regard to the angular velocity $\Omega$ and the strange quark mass $m_{s}$ :

$$
\frac{1}{i \tilde{D}} \simeq \frac{1}{\omega+i H}-\frac{1}{\omega+i H} \Omega \frac{1}{\omega+i H}-\frac{1}{\omega+i H} \gamma_{4} R^{\dagger}(t) \hat{m} R(t) \frac{1}{\omega+i H} .
$$

The transition matrix element of the hadronic weak current given by Eq.(3) can be related to the correlation function

$$
\left\langle 0\left|J_{B_{1}}\left(\vec{x}, \frac{T}{2}\right) \bar{\psi} \hat{\Gamma} \hat{O} \psi J_{B_{2}}^{\dagger}\left(\vec{y},-\frac{T}{2}\right)\right| 0\right\rangle
$$

at large Euclidean time T. $\hat{\Gamma}$ and $\hat{O}$ are abbreviations for the corresponding spin and flavor operators. The $J_{B}$ denotes the baryon current which is constructed from $N_{c}$ quark fields

$$
J_{B}=\frac{1}{N_{c} !} \varepsilon^{i_{1} \ldots i_{N_{c}}} \Gamma_{S S_{3} I I_{3} Y}^{\alpha_{1} \ldots \alpha_{N_{c}}} \psi_{\alpha_{1} i_{1}} \ldots \psi_{\alpha_{N_{c}} i_{N_{c}}}
$$

$\alpha_{1} \ldots \alpha_{N_{c}}$ are spin-isospin indices, $i_{1} \ldots i_{N_{c}}$ are color indices, and the matrices $\Gamma_{S S_{3} I I_{3} Y}^{\alpha_{1} \ldots \alpha_{N_{c}}}$ are taken to endow the corresponding current with the quantum numbers $S S_{3} I I_{3} Y$. The $J_{B}\left(J_{B}^{\dagger}\right)$ 
plays a role of annihilating (creating) the baryon state at large $T$. The rotational $1 / N_{c}$ and linear $m_{s}$ corrections being taken into account as shown in Eq.(16), the relevant transition matrix elements can be written as follows:

$$
\begin{aligned}
\left(f_{1}+f_{2}\right)^{\left(B_{1} \rightarrow B_{2}\right)} & =w_{1}\left\langle B_{2}\left|D_{X 3}^{(8)}\right| B_{1}\right\rangle+w_{2} d_{p q 3}\left\langle B_{2}\left|D_{X p}^{(8)} \hat{S}_{q}\right| B_{1}\right\rangle+\frac{w_{3}}{\sqrt{3}}\left\langle B_{2}\left|D_{X 8}^{(8)} \hat{S}_{3}\right| B_{1}\right\rangle \\
& +m_{s}\left[\frac{w_{4}}{\sqrt{3}} d_{p q 3}\left\langle B_{2}\left|D_{X p}^{(8)} D_{8 q}^{(8)}\right| B_{1}\right\rangle+w_{5}\left\langle B_{2}\left|\left(D_{X 3}^{(8)} D_{88}^{(8)}+D_{X 8}^{(8)} D_{83}^{(8)}\right)\right| B_{1}\right\rangle\right. \\
& \left.+w_{6}\left\langle B_{2}\left|\left(D_{X 3}^{(8)} D_{88}^{(8)}-D_{X 8}^{(8)} D_{83}^{(8)}\right)\right| B_{1}\right\rangle\right]
\end{aligned}
$$

for the transition magnetic moments $\left(f_{1}(0)+f_{2}(0)\right)^{\left(B_{1} \rightarrow B_{2}\right)}$ and

$$
\begin{aligned}
g_{1}^{\left(B_{1} \rightarrow B_{2}\right)} & =a_{1}\left\langle B_{2}\left|D_{X 3}^{(8)}\right| B_{1}\right\rangle+a_{2} d_{p q 3}\left\langle B_{2}\left|D_{X p}^{(8)} \hat{S}_{q}\right| B_{1}\right\rangle+\frac{a_{3}}{\sqrt{3}}\left\langle B_{2}\left|D_{X 8}^{(8)} \hat{S}_{3}\right| B_{1}\right\rangle \\
& +m_{s}\left[\frac{a_{4}}{\sqrt{3}} d_{p q 3}\left\langle B_{2}\left|D_{X p}^{(8)} D_{8 q}^{(8)}\right| B_{1}\right\rangle+a_{5}\left\langle B_{2}\left|\left(D_{X 3}^{(8)} D_{88}^{(8)}+D_{X 8}^{(8)} D_{83}^{(8)}\right)\right| B_{1}\right\rangle\right. \\
& \left.+a_{6}\left\langle B_{2}\left|\left(D_{X 3}^{(8)} D_{88}^{(8)}-D_{X 8}^{(8)} D_{83}^{(8)}\right)\right| B_{1}\right\rangle\right],
\end{aligned}
$$

for the transition axial constants $g_{1}^{\left(B_{1} \rightarrow B_{2}\right)}(0)$. The induced pseudotensor coupling constants $g_{2}^{\left(B_{1} \rightarrow B_{2}\right)}$ are expressed by

$$
\frac{g_{2}^{\left(B_{1} \rightarrow B_{2}\right)}}{M_{B_{1}}}=4 m_{s}\left(\beta_{1} i f_{a b 3}+\beta_{2} i \varepsilon_{a b 3}\right) \cdot\left\langle B_{2}\left|D_{X a} D_{8 b}\right| B_{1}\right\rangle .
$$

The parameters $w_{i}, a_{i}$ and $\beta_{j}$ depend on dynamics of the chiral soliton. As for the expressions for $w_{i}$ and $a_{i}$, one can find them in Ref. [29] and Ref. [16], respectively. $\beta_{1}$ and $\beta_{2}$ can be written explicitly as 30,31

$$
\begin{aligned}
\beta_{1}+\beta_{2} & =\frac{i N_{c}}{24 \sqrt{3}} \int \frac{d \omega}{2 \pi} \operatorname{Sp}\left(\frac{1}{\omega+i H} \gamma_{4} \tau^{i} \frac{1}{\omega+i H} i \varepsilon_{i j k} \tau^{j} x^{k} \gamma_{5}\right), \\
\beta_{1} & =-\frac{i N_{c}}{12 \sqrt{3}} \int \frac{d \omega}{2 \pi} \operatorname{Sp}\left(\left\{\frac{1}{\omega+i H} \gamma_{4}, \frac{1}{\omega+i H_{0}}\right\} \gamma_{5} \vec{x} \cdot \vec{\tau}\right) .
\end{aligned}
$$

The remarkable feature of the soliton picture of the baryons is that the singlet axial charge of the nucleon $g_{A}^{(0)}$ is expressed in terms of the same parameters $a_{i}$ in Eq.(20):

$$
g_{A}^{(0)}=\frac{1}{2} a_{3}+\sqrt{3} m_{s}\left(a_{5}-a_{6}\right)
$$

Hence, in this picture the value of $g_{A}^{(0)}$ can be extracted by fitting the data on semileptonic decays without resorting to those on polarized deep inelastic scattering (see the next section for the analysis of the SU(3)-symmetric case). With SU(3) symmetry explicitly broken by $m_{s}$, the collective Hamiltonian is no more $\mathrm{SU}(3)$-symmetric. The pure octet states are mixed with the higher representations such as anti-decuplet states. Therefore, the baryon wave function with spin $S=1 / 2$ requires the modification due to the strange quark mass $\left(m_{s}\right)$. Since we treat $m_{s}$ perturbatively up to the first order, the collective wave function of the baryon octet can be written as 


$$
\Psi_{B}(R)=\Psi_{B}^{(8)}(R)+m_{\mathrm{s}} c \frac{B}{10} \Psi_{B}^{(\overline{10})}(R)+m_{\mathrm{s}} c_{27}^{B} \Psi_{B}^{(27)}(R)
$$

where

$$
c \frac{B}{10}=\frac{\sqrt{5}}{15}\left(\sigma-r_{1}\right)\left[\begin{array}{l}
1 \\
0 \\
1 \\
0
\end{array}\right] I_{2}, \quad c_{27}^{B}=\frac{1}{75}\left(3 \sigma+r_{1}-4 r_{2}\right)\left[\begin{array}{c}
\sqrt{6} \\
3 \\
2 \\
\sqrt{6}
\end{array}\right] I_{2}
$$

in the basis of $[N, \Lambda, \Sigma, \Xi]$. Here, $B$ denotes the $\mathrm{SU}(3)$ octet baryons with the spin $1 / 2$. The constant $\sigma$ is related to the nucleon sigma term $\Sigma=\bar{m}\langle N|\bar{u} u+\bar{d} d| N\rangle=3 / 2 \bar{m} \sigma$ and $r_{i}$ designates $K_{i} / I_{i}$, where $K_{i}$ stands for the anomalous moments of inertia defined in Ref. [32]. The collective wave function can be explicitly written in terms of the $\mathrm{SU}(3)$ Wigner $D^{(\mu)}$ function:

$$
\Psi_{B}^{(\mu)}=(-)^{S_{3}-1 / 2} \sqrt{\operatorname{dim}(\mu)}\left[D_{\left(Y T T_{3}\right)\left(-1 S S_{3}\right)}^{(\mu)}\right]^{*} .
$$

Hence, we have two different contributions of SU(3) symmetry breaking: One from the effective Lagrangian and the other from the wave function corrections. All contributions of $\mathrm{SU}(3)$ symmetry breaking are kept in linear order of $m_{s}$. Apart from these two contributions, we shall see in the next section that in the case of $f_{2}(0)$ the mass differences between octet baryons come into play. Hence, on the whole, we have three different sources for the $\mathrm{SU}(3)$ symmetry breaking in the present model.

\section{SEMILEPTONIC DECAY CONSTANTS IN THE $\chi$ QSM}

\section{A. Exact SU(3) symmetry}

In exact $\mathrm{SU}(3)$ symmetry, the vector coupling constants $f_{1}(0)$ and $f_{2}(0)$ can be simply expressed in terms of the anomalous magnetic moments of the proton and neutron. Similarly, $g_{1}(0)$ in all decay modes can be parametrized in terms of two $\mathrm{SU}(3)$-invariant constants $F$ and $D$ [33:

$$
g_{1}^{\left(B_{1} \rightarrow B_{2}\right)}=F C_{F}^{B_{1} \rightarrow B_{2}}+D C_{D}^{B_{1} \rightarrow B_{2}},
$$

where $C_{F}^{B_{1} \rightarrow B_{2}}$ and $C_{D}^{B_{1} \rightarrow B_{2}}$ are SU(3) Clebsch-Gordan coefficients that appear when an octet operator is sandwiched between octet states. The superscript refers to the hadrons involved and subscripts $F$ and $D$ denote the antisymmetric and symmetric parts. We list the expressions for $f_{1}(0), f_{2}(0)$ and $g_{1}(0)$ in Table I 4 .

The pseudotensor coupling constants $g_{2}(0)$ are all predicted to be zero in exact $\mathrm{SU}(3)$ symmetry because of $G$-parity. In fact, it can be shown that $g_{2}(0)$ vanish in the present model. To do so, it is of great use to introduce a transformation

\footnotetext{
${ }^{1}$ Note that the signs in the $\Lambda \rightarrow p$, and $\Sigma^{-} \rightarrow n$ modes are different from Ref. 33]. However, the ratios $f_{2}(0) / f_{1}(0)$ and $g_{1}(0) / f_{1}(0)$ are not affected. We have employed the phase convention à la De Swart [34,35].
} 


$$
G_{5}=\tau_{2} C \gamma_{5}
$$

where $C$ is the operator of charge conjugation: $C^{-1} \gamma_{\mu} C=-\gamma_{\mu}^{T}$. Under this transformation, the one-body Dirac Hamiltonian, Dirac and Pauli matrices are respectively changed as follows:

$$
\begin{aligned}
G_{5}^{-1} H G_{5} & =H^{T}, \\
G_{5}^{-1} \gamma_{\alpha} G_{5} & =\gamma_{\alpha}^{T}, \text { for } \alpha=1, \cdots, 5 \\
G_{5}^{-1} \tau_{a} G_{5} & =-\tau_{a}^{T} .
\end{aligned}
$$

The pertinent trace for the leading order contribution to $g_{2}(0)$ can be written as

$$
\operatorname{tr}\left[\left\langle\vec{x}\left|\frac{1}{\omega+i H} \gamma_{5} \tau_{a} x_{i}\right| \vec{x}\right\rangle\right]
$$

Utilizing the $G_{5}$ transformation and the properties of the trace $\operatorname{tr}\left(M^{T}\right)=\operatorname{tr}(M)$ and $\operatorname{tr}\left(W M W^{-1}\right)=\operatorname{tr}(M)$, we can show that the trace of the leading contribution vanishes:

$$
\begin{aligned}
\operatorname{tr}\left[\left\langle\vec{x}\left|\frac{1}{\omega+i H} \gamma_{5} \tau_{a} x_{i}\right| \vec{x}\right\rangle\right] & =\operatorname{tr}\left[\left\langle\vec{x}\left|G_{5}^{-1} \frac{1}{\omega+i H} \gamma_{5} \tau_{a} x_{i} G_{5}\right| \vec{x}\right\rangle\right]^{T} \\
& =-\operatorname{tr}\left[\left\langle\vec{x}\left|\frac{1}{\omega+i H} \gamma_{5} \tau_{a} x_{i}\right| \vec{x}\right\rangle\right] .
\end{aligned}
$$

Similarly we can prove that the $1 / N_{c}$ rotational corrections to $g_{2}(0)$ also disappear.

In exact SU(3) symmetry, $g_{1}^{\left(B_{1} \rightarrow B_{2}\right)}$ can be written in terms of three independent dynamic quantities $a_{i}$ calculable in the present model:

$$
g_{1}^{\left(B_{1} \rightarrow B_{2}\right)}=a_{1}\left\langle B_{2}\left|D_{X 3}^{(8)}\right| B_{1}\right\rangle+a_{2} d_{p q 3}\left\langle B_{2}\left|D_{X p}^{(8)} \hat{S}_{q}\right| B_{1}\right\rangle+\frac{a_{3}}{\sqrt{3}}\left\langle B_{2}\left|D_{X 8}^{(8)} \hat{S}_{3}\right| B_{1}\right\rangle .
$$

As for the process $n \rightarrow p e^{-} \bar{\nu}, g_{1}^{(n \rightarrow p)}$ becomes just the axial coupling constant $g_{A}^{(3)}$. After some straightforward manipulation [16, we end up with the expression for $g_{A}^{(3)}$,36, 37]

$$
g_{A}^{(3)}=\frac{7}{30}\left(-a_{1}+\frac{1}{2} a_{2}+\frac{1}{14} a_{3}\right) .
$$

We can also obtain the singlet axial coupling constant $g_{A}^{(0)}$

$$
g_{A}^{(0)}=\frac{1}{2} a_{3}
$$

It is of great interest to see how the $\chi$ QSM plays an interpolating role between the Skyrme model and the NRQM. In the limit of the NRQM the dynamic quantities $a_{i}$ in Eq.(33) are respectively 38,39

$$
a_{1}=-5, \quad a_{2}=4, \quad a_{3}=2,
$$

which give the correct values of $g_{A}^{(3)}$ and $g_{A}^{(0)}$ in the NRQM, i.e. $g_{A}^{(3)}=5 / 3$ and $g_{A}^{(0)}=1$. The ratio $F / D$ then can be written in terms of $a_{i}$ 


$$
\frac{F}{D}=\frac{5}{9}\left(\frac{-a_{1}+\frac{1}{2} a_{2}+\frac{1}{2} a_{3}}{-a_{1}+\frac{1}{2} a_{2}-\frac{1}{6} a_{3}}\right)
$$

In the limit of the Skyrme model, i.e. when the size of the soliton is very large [25], $g_{A}^{(0)}$ approaches zero or $a_{3}$ vanishes. Hence, the $F / D$ ratio becomes obviously $5 / 9$ in this limit. This result is exactly the same as what was obtained by Bijnens et al. 40] and Chemtob 41]. On the other hand, in the limit of the NRQM, i.e. in the limit of zero soliton size, the present model gives the value of $F / D=2 / 3$ which is exactly the same value as that of the SU(6) NRQM. The present model predicts $F / D$ to be 0.61 - correspondingly, we have $g_{A}^{(0)}=0.36$ 37 - which lies between the value from the Skyrme model (5/9) and that from the NRQM (2/3). The $\chi$ QSM shows here again interpolation between the Skyrme model and the NRQM [25,26]. Notably, the smallness of the singlet axial charge $g_{A}^{(0)}$ is directly related to the fact that $F / D$ does not deviate much from that of the Skyrme model (5/9), where $g_{A}^{(0)}$ is known to be zero [42. Using Eqs. 34, 35, 37), we can express the singlet axial constant $g_{A}^{(0)}$ in terms of the $F / D$ ratio and $g_{A}^{(3)}$ :

$$
g_{A}^{(0)}=\frac{9 g_{A}^{(3)}}{1+F / D}\left(\frac{F}{D}-\frac{5}{9}\right) .
$$

Substituting the value of $F / D=0.582$ obtained in a recent analysis $\left[13\right.$ and $g_{A}^{(3)}=1.26$, one gets $g_{A}^{(0)}=0.19$.

The $g_{1}$ is normally determined in experiments with $g_{2}$ assumed to be zero. However, Hsueh et al. [5] extracted for the first time the induced pseudotensor coupling constant $g_{2}$ in $\Sigma^{-} \rightarrow n e^{-} \bar{\nu}$ decay. This new experimental results give a reduced value for $g_{1}(0)$ : Instead of $g_{1}(0)=0.328 \pm 0.019$ and $g_{2}=0, g_{1}(0)=0.20 \pm 0.08$ and $g_{2}(0)=-0.56 \pm 0.37$ are obtained. These results are remarkable, since they indicate that the effect of SU(3) symmetry breaking might play an important role in baryon semileptonic decays. In fact, these new experimental results have triggered discussions about the effect of $\mathrm{SU}(3)$ symmetry breaking in hyperon semileptonic decays [11,13. Hence, we need to consider the explicit $\mathrm{SU}(3)$ symmetry breaking.

\section{B. SU(3) symmetry breaking effects}

As we have shown in section II, the strange quark mass $m_{s}$ provides the effect of $\mathrm{SU}(3)$ symmetry breaking in two different forms: One from the effective action and the other from the wave function corrections. Apart from these two contributions, the mass difference between baryon states must be considered in the case of $f_{2}(0)$.

By switching on $\mathrm{SU}(3)$ symmetry breaking to the first order in $m_{s}$, we obtain the expressions for $f_{2}(0)$ and $g_{1}(0)$ deviating from those listed in Table I. It should be noted that by the Ademollo-Gatto theorem [43] $f_{1}(0)$ do not get any contribution from linear $m_{s}$ corrections. We choose the combination of the magnetic moments in which all corrections from $1 / N_{c}$ are cancelled to avoid ambiguity arising from the relations between the hyperon magnetic moments 15]. Hence we get the unambiguous relations between hyperon magnetic moments and off-diagonal matrix elements: 


$$
\begin{aligned}
f_{2}^{(n \rightarrow p)}(0) & =\frac{1}{2}\left(\kappa_{p}-\kappa_{n}\right), \\
f_{2}^{\left(\Sigma^{-} \rightarrow \Sigma^{0}\right)}(0) & =\frac{M_{\Sigma}}{2 M_{N}} \frac{1}{\sqrt{2}}\left(\kappa_{\Sigma^{+}}-\kappa_{\Sigma^{-}}\right), \\
f_{2}^{\left(\Sigma^{ \pm} \rightarrow \Lambda\right)}(0) & =\mp \frac{M_{\Sigma}}{\sqrt{2} M_{N}} \mu_{\Sigma^{0} \Lambda}, \\
\mu_{\Sigma^{0} \Lambda} & =\frac{1}{\sqrt{3}}\left(-\kappa_{n}+\frac{1}{4}\left(\kappa_{\Sigma^{+}}+\kappa_{\Sigma^{-}}\right)-\kappa_{\Xi^{0}}+\frac{3}{2} \kappa_{\Lambda}\right), \\
f_{2}^{(\Lambda \rightarrow p)}(0) & =\frac{M_{\Lambda}}{2 \sqrt{6} M_{N}}\left(\kappa_{n}+\frac{5}{2} \kappa_{p}+\frac{1}{2} \kappa_{\Sigma^{-}}-3 \kappa_{\Lambda}-\kappa_{\Xi^{-}}\right), \\
f_{2}^{\left(\Sigma^{-} \rightarrow n\right)}(0) & =\frac{M_{\Sigma}}{2 M_{N}}\left(\kappa_{n}+\frac{1}{2} \kappa_{p}-\kappa_{\Sigma^{-}}-\frac{1}{2} \kappa_{\Sigma^{+}}\right), \\
f_{2}^{\left(\Xi^{-} \rightarrow \Lambda\right)}(0) & =\frac{M_{\Xi}}{2 \sqrt{6} M_{N}}\left(\kappa_{p}-\frac{1}{2} \kappa_{\Sigma^{+}}+3 \kappa_{\Lambda}-\kappa_{\Xi^{0}}-\frac{5}{2} \kappa_{\Xi^{-}}\right), \\
f_{2}^{\left(\Xi^{-} \rightarrow \Sigma^{0}\right)}(0) & =\frac{M_{\Xi}}{2 \sqrt{2} M_{N}}\left(\kappa_{\Sigma^{+}}+\frac{1}{2} \kappa_{\Sigma^{-}}-\kappa_{\Xi^{0}}-\frac{1}{2} \kappa_{\Xi^{-}}\right),
\end{aligned}
$$

where $\kappa_{B}$ is the anomalous magnetic moment corresponding to the baryon $B$. These relations have corrections of order $\mathcal{O}\left(m_{s}^{2}\right)$ and $\mathcal{O}\left(m_{s} / N_{c}\right)$ which are assumed to be small. In exact $\mathrm{SU}(3)$ symmetry these relations reduce again to the $\mathrm{SU}(3)$ relations shown in Table I. In Table II we compare the results with SU(3) symmetry breaking to those in SU(3) symmetry. The experimental data for $f_{2}(0)$ are taken from Ref. [6] for $\Lambda \rightarrow p e^{-} \bar{\nu}$ and from Ref. [5] for $\Sigma^{-} \rightarrow n e^{-} \bar{\nu}$. Incorporating experimental values for magnetic moments taken from 444] into formulae in Table I and those in Eq.(39), respectively, we obtain the $f_{2}(0) / f_{1}(0)$ ratios for seven different channels 2. Let us first compare the first two columns. Apart from the $|\Delta S|=0$ modes for which the effect of SU(3) symmetry breaking is observed in around $10 \%$, we can see the comparably large effect of the SU(3) symmetry breaking. Considering the $\mathrm{SU}(3)$ symmetry breaking inherent already in experimental magnetic moments, one can say that the effect of $\mathrm{SU}(3)$ symmetry breaking is even larger. From the comparison of the third and fourth columns, we can find the effects of $\mathrm{SU}(3)$ symmetry breaking. The effects on $f_{2}(0) / f_{1}(0)$ are noticeably large in almost every decay mode. In particular, the deviation from $\mathrm{SU}(3)$ symmetry appearing in the $\Sigma^{-} \rightarrow n e^{-} \bar{\nu}$ mode is remarkable. Indeed, the effects of $\mathrm{SU}(3)$ symmetry breaking pull $f_{2}(0) / f_{1}(0)\left(\Sigma^{-} \rightarrow n\right)$ drastically down from its $\mathrm{SU}(3)$ symmetric value, so that it turns out to be in good agreement with the data [5].

The expressions of $g_{1}(0)$ for the case of SU(3) symmetry breaking can be obtained similarly to the SU(3) symmetric case (see Table I). For convenience, we define the baryonic axial constants as follows:

$$
g_{A}^{B}=\left\langle B\left|\left(g_{A}^{(3)}+\frac{1}{\sqrt{3}} g_{A}^{(8)}\right)\right| B\right\rangle .
$$

\footnotetext{
${ }^{2}$ Note that though we use the SU(3) symmetric expressions to obtain the first column in Table II the results nevertheless include a part of the $\mathrm{SU}(3)$ symmetry breaking through the experimental data. However, by doing that we can see at least the effect of SU(3) symmetry breaking within the framework of the $\chi$ QSM.
} 
Then we can write the expressions for $g_{1}(0)\left(B_{1} \rightarrow B_{2}\right)$ similar to Eq.(39):

$$
\begin{aligned}
g_{1}^{(n \rightarrow p)}(0) & =\frac{1}{2}\left(g_{A}^{p}-g_{A}^{n}\right), \\
g_{1}^{\left(\Sigma^{-} \rightarrow \Sigma^{0}\right)}(0) & =\frac{1}{2 \sqrt{2}}\left(g_{A}^{\Sigma^{+}}-g_{A}^{\Sigma^{-}}\right), \\
g_{1}^{\left(\Sigma^{ \pm} \rightarrow \Lambda\right)}(0) & =\mp \frac{1}{2} \sqrt{\frac{2}{3}}\left(-g_{A}^{n}+\frac{1}{2} g_{A}^{\Sigma^{0}}-g_{A}^{\Xi^{0}}+\frac{3}{2} g_{A}^{\Lambda}\right), \\
g_{1}^{(\Lambda \rightarrow p)}(0) & =\frac{1}{2 \sqrt{6}}\left(g_{A}^{n}+\frac{5}{2} g_{A}^{p}+\frac{1}{2} g_{A}^{\Sigma^{-}}-3 g_{A}^{\Lambda}-g_{A}^{\Xi^{-}}\right), \\
g_{1}^{\left(\Sigma^{-} \rightarrow n\right)}(0) & =\frac{1}{2}\left(g_{A}^{n}+\frac{1}{2} g_{A}^{p}-g_{A}^{\Sigma^{-}}-\frac{1}{2} g_{A}^{\Sigma^{+}}\right), \\
g_{1}^{\left(\Xi^{-} \rightarrow \Lambda\right)}(0) & =\frac{1}{2 \sqrt{6}}\left(g_{A}^{p}-\frac{1}{2} g_{A}^{\Sigma^{+}}+3 g_{A}^{\Lambda}-g_{A}^{\Xi^{0}}-\frac{5}{2} g_{A}^{\Xi^{-}}\right), \\
g_{1}^{\left(\Xi^{-} \rightarrow \Sigma^{0}\right)}(0) & =\frac{1}{2 \sqrt{2}}\left(g_{A}^{\Sigma^{+}}+\frac{1}{2} g_{A}^{\Sigma^{-}}-g_{A}^{\Xi^{0}}-\frac{1}{2} g_{A}^{\Xi^{-}}\right),
\end{aligned}
$$

Making use of Eq.(20), we obtain the sum rule between six different decay modes

$$
g_{1}^{(n \rightarrow p)}=\frac{1}{2}\left(\sqrt{2} g_{1}^{\left(\Sigma^{-} \rightarrow \Sigma^{0}\right)}+\sqrt{6} g_{1}^{\left(\Sigma^{+} \rightarrow \Lambda\right)}-\sqrt{6} g_{1}^{(\Lambda \rightarrow p)}+g_{1}^{\left(\Sigma^{-} \rightarrow n\right)}-2 \sqrt{2} g_{1}^{\left(\Xi^{-} \rightarrow \Sigma^{0}\right)}\right) .
$$

In order to verify Eq.(42), more accurate experimental data are required than presently available. In the $\mathrm{SU}(3)$ limit the right hand side of Eq. (42) becomes $D+F$ in accordance with the Cabibbo theory 47 .

In Table III, the results of $g_{1}(0) / f_{1}(0)$ with $\mathrm{SU}(3)$ symmetry breaking are compared to those in SU(3) symmetry. The effect of $\mathrm{SU}(3)$ symmetry breaking is measured in $5 \sim 10 \%$, which is not that strong. Compared to the case of $f_{2}(0) / f_{1}(0)$, the effect of SU(3) symmetry breaking is rather soft. This is partly due to the fact that in the axial channel the mass differences do not come into play, which is somewhat in line with the argument of Ref. [13. The results predicted by the $\chi$ QSM are in good agreement with the experimenta data 44 within about $15 \%$ which is a typical predictive power of the model. In particular, the results agree with the data remarkably in $|\Delta S|=1$ channels.

It is also interesting to see that in the present model the ratio of $g_{1} / f_{1}$ between $\Lambda \rightarrow p e^{-} \bar{\nu}$ decay and $\Sigma^{-} \rightarrow n e^{-} \bar{\nu}$ decay is well reproduced

$$
\frac{g_{1} / f_{1}\left(\Lambda \rightarrow p e^{-} \bar{\nu}\right)}{g_{1} / f_{1}\left(\Sigma^{-} \rightarrow n e^{-} \bar{\nu}\right)}=-2.28(\operatorname{Exp}:-2.11 \pm 0.15)
$$

It was pointed out that this ratio is a priori constrained to -3 in quark models with $\mathrm{SU}(6)$ symmetry 48,49], which is noticeably larger than the experimental value.

It is also interesting to compare the present results with those from the Skyrme model with vector mesons [50]. Ref. [50] presented the $g_{1} / f_{1}$ ratio in five different channels. Except for the $\Lambda \rightarrow p$ mode, the present model seems to be far better than Ref. [50]. For example, we obtain the $\left|g_{1} / f_{1}\right|=0.31$ for the $\Sigma^{-} \rightarrow n$ mode comparable to the experimental data $0.34 \pm 0.017$, while Ref. [50] yields 0.24 .

From Eq.(21), we can obtain the ratio $g_{2}(0) / f_{1}(0)$ in terms of $\beta_{1}$ and $\beta_{2}$. In Table IV, the expressions and numerical results for them are listed. Numerically, $\beta_{2}$ is much larger than 
$\beta_{1}$, which explains why the $g_{2} / f_{1}$ for the $\Xi^{-} \rightarrow \Sigma^{0}$ mode turns out to be much greater than those for the other modes. Our results are compared with results of an $\mathrm{SU}(6)$ relativistic quark model [51] as well as with those of a light-front relativistic quark model [49]. We see that our results are close - within factor of 2 - to the results of Ref. [49], whereas differ by almost order of magnitude from those of Ref. [51].

\section{SUMMARY AND CONCLUSION}

The aim of the present work has been to investigate baryon semileptonic decays within the framework of the chiral quark-soliton model $(\chi \mathrm{QSM})$. In particular, the role of $\mathrm{SU}(3)$ symmetry breaking in baryon semileptonic decays have been discussed. Based on the recent studies on the octet magnetic moments and axial vector constants, we have obtained the ratios $f_{2} / f_{1}$ and $g_{1} / f_{1}$ without and with $\mathrm{SU}(3)$ symmetry breaking, respectively. In exact $\mathrm{SU}(3)$ symmetry, we have shown that $g_{2}$ vanishes in the present model, as it should be. We have discussed also that for the $F / D$ ratio the $\chi$ QSM (0.61) interpolates the Skyrme model $(5 / 9)$ and the NRQM $(2 / 3)$.

It was found that the effect of $\mathrm{SU}(3)$ symmetry breaking differs in different channels and modes. In general, $\mathrm{SU}(3)$ symmetry breaking contributes strongly to the ratio of the vector coupling constants $f_{2}(0) / f_{1}(0)$ while it does not much to the ratio $g_{1}(0) / f_{1}(0)$. In addition, we have evaluated the ratio $g_{2} / f_{1}$. This is the first calculation of the $g_{2} / f_{1}$ in soliton models. The results come out to be small except for the $\Xi^{-} \rightarrow \Sigma^{0}$ mode. Due to lack of experimental data, the values of $g_{2}(0)$ we calculated are predictions.

\section{ACKNOWLEDGMENT}

This work has partly been supported by the BMBF, the DFG and the COSY-Project (Jülich). M.V.P. and M.P. have been supported by Alexander von Humboldt Stiftung. 


\section{TABLES}

TABLE I. The expressions of $f_{1}(0), f_{2}(0)$ and $g_{1}(0)$ in exact $\mathrm{SU}(3)$ symmetry. The $\kappa_{p}$ and the $\kappa_{n}$ denote the anomalous magnetic moments of the proton and the neutron, respectively.

\begin{tabular}{cccc}
\hline \hline Decay mode & $f_{1}(0)$ & $f_{2}(0)$ & $g_{1}(0)$ \\
\hline$n \rightarrow p$ & 1 & $\frac{1}{2}\left(\kappa_{p}-\kappa_{n}\right)$ & $F+D$ \\
$\Sigma^{-} \rightarrow \Sigma^{0}$ & $\sqrt{2}$ & $\frac{1}{\sqrt{2}}\left(\kappa_{p}+\frac{1}{2} \kappa_{n}\right)$ & $\sqrt{2} F$ \\
$\Sigma^{ \pm} \rightarrow \Lambda$ & 0 & $\pm \sqrt{\frac{3}{8}} \kappa_{n}$ & $\mp \sqrt{\frac{2}{3}} D$ \\
$\Lambda \rightarrow p$ & $\sqrt{\frac{3}{2}}$ & $\frac{1}{2} \sqrt{\frac{3}{2}} \kappa_{p}$ & $\sqrt{\frac{3}{2}}(F+D / 3)$ \\
$\Sigma^{-} \rightarrow n$ & 1 & $\frac{1}{2}\left(\kappa_{p}+2 \kappa_{n}\right)$ & $F-D$ \\
$\Xi^{-} \rightarrow \Lambda$ & $\sqrt{\frac{3}{2}}$ & $\frac{1}{2} \sqrt{\frac{3}{2}}\left(\kappa_{p}+\kappa_{n}\right)$ & $\sqrt{\frac{3}{2}}(F-D / 3)$ \\
$\Xi^{-} \rightarrow \Sigma^{0}$ & $\frac{1}{\sqrt{2}}$ & $\frac{1}{2 \sqrt{2}}\left(\kappa_{p}-\kappa_{n}\right)$ & $\sqrt{\frac{1}{2}}(F+D)$ \\
\hline \hline
\end{tabular}

TABLE II. The results of $f_{2}(0) / f_{1}(0)$. The column under $\mathrm{SU}(3)_{\text {sym }}$ lists the case of exact $\mathrm{SU}(3)$ symmetry with the experimental data of $\kappa_{p}$ and $\kappa_{n}$ while the next column shows the case of broken $\mathrm{SU}(3)$ symmetry by $m_{s}$ with the experimental data of $\kappa_{B}$ 44. The third column presents the numerical results of $f_{2}(0)$ in the $\chi \mathrm{QSM}$ with the constituent quark mass $M=420 \mathrm{MeV}$, while the fourth one shows those of $f_{2}(0)$ in the $\chi \mathrm{QSM}$ with the same constituent quark mass. The experimental data are taken from Ref. [6] for $\Lambda \rightarrow p e^{-} \bar{\nu}$ and from Ref. [5] for $\Sigma^{-} \rightarrow n e^{-} \bar{\nu}$.

\begin{tabular}{cccccc}
\hline \hline decay mode & $\mathrm{SU}(3)_{\text {sym }}$ & $\mathrm{SU}(3)_{\mathrm{br}}$ & $\chi \mathrm{QSM}_{\text {sym }}$ & $\chi \mathrm{QSM}_{\mathrm{br}}$ & $\operatorname{Exp}$ \\
\hline$n \rightarrow p$ & 1.853 & 1.853 & 1.41 & 1.58 & -- \\
$\Sigma^{-} \rightarrow \Sigma^{0}$ & 0.418 & $0.516 \pm 0.012$ & 0.25 & 0.43 & -- \\
$\Sigma^{-} \rightarrow \Lambda$ & $1.435^{\mathrm{a}}$ & $1.625 \pm 0.011^{\mathrm{a}}$ & $0.95^{\mathrm{a}}$ & $1.33^{\mathrm{a}}$ & -- \\
$\Lambda \rightarrow p$ & 0.896 & $0.787 \pm 0.004$ & 0.64 & 0.74 & $0.15 \pm 0.30$ \\
$\Sigma^{-} \rightarrow n$ & -1.017 & $-1.010 \pm 0.016$ & -0.92 & -1.18 & $-0.96 \pm 0.07 \pm 0.13$ \\
$\Xi^{-} \rightarrow \Lambda$ & -0.060 & $-0.093 \pm 0.006$ & -0.14 & -0.18 & -- \\
$\Xi^{-} \rightarrow \Sigma^{0}$ & 1.853 & $1.725 \pm 0.011$ & 1.41 & 2.06 & -- \\
\hline \hline
\end{tabular}

anstead of $f_{2} / f_{1}$, we list $\sqrt{\frac{3}{2}} f_{2}$. 
TABLE III. The results of $g_{1}(0) / f_{1}(0)$. The column under $\chi \mathrm{QSM}_{\mathrm{sym}}$ lists the numerical results in exact $\mathrm{SU}(3)$ symmetry while in the next column shows the case of broken $\mathrm{SU}(3)$ symmetry by $m_{s}$. The constituent quark mass $M=420 \mathrm{MeV}$ is used. Most experimental data are taken from Particle Data Group [44]. The data for the $\Sigma^{-} \rightarrow \Lambda$ mode are taken from [45] while that for the $\Xi^{-} \rightarrow \Sigma^{0}$ mode are from 46 .

\begin{tabular}{cccc}
\hline \hline decay mode & $\chi \mathrm{QSM}_{\text {sym }}$ & $\chi \mathrm{QSM}_{\mathrm{br}}$ & $\operatorname{Exp}$ \\
\hline$n \rightarrow p$ & 1.33 & 1.42 & $1.2573 \pm 0.0028$ \\
$\Sigma^{-} \rightarrow \Sigma^{0}$ & 0.50 & 0.55 & -- \\
$\Sigma^{-} \rightarrow \Lambda$ & $0.83^{\mathrm{a}}$ & $0.91^{\mathrm{a}}$ & $0.720 \pm 0.020^{\mathrm{a}}$ \\
$\Lambda \rightarrow p$ & 0.78 & 0.73 & $0.718 \pm 0.015$ \\
$\Sigma^{-} \rightarrow n$ & -0.33 & -0.31 & $-0.340 \pm 0.017$ \\
$\Xi^{-} \rightarrow \Lambda$ & 0.23 & 0.22 & $0.25 \pm 0.05$ \\
$\Xi^{-} \rightarrow \Sigma^{0}$ & 1.33 & 1.29 & $1.25_{-0.16}^{+0.14}$ \\
\hline \hline
\end{tabular}

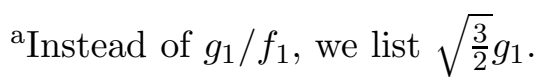

TABLE IV. The induced pseudotensor coupling constants ratio $g_{2}(0) / f_{1}(0)$. The results are compared with Refs. [49,51.

\begin{tabular}{ccccc}
\hline \hline decay mode & expression & $\chi$ QSM & Schlumpf & Kellett \\
\hline$n \rightarrow p$ & 0 & 0 & 0 & 0.29 \\
$\Sigma^{-} \rightarrow \Sigma^{0}$ & 0 & 0 & 0 & -- \\
$\Sigma^{-} \rightarrow \Lambda$ & $-\frac{4 m_{s}}{5 \sqrt{3}} M_{\Sigma^{-}} \beta_{2}{ }^{\mathrm{a}}$ & $-0.029^{\mathrm{a}}$ & $0^{\mathrm{a}}$ & $0.18^{\mathrm{a}}$ \\
$\Lambda \rightarrow p$ & $\frac{8 m_{s}}{5 \sqrt{3}} M_{\Lambda}\left(\beta_{1}+\frac{1}{2} \beta_{2}\right)$ & 0.046 & 0.023 & 0.25 \\
$\Sigma^{-} \rightarrow n$ & $-\frac{4 m_{s}}{15 \sqrt{3}} M_{\Sigma^{-}}\left(3 \beta_{1}+\beta_{2}\right)$ & -0.020 & -0.007 & -0.09 \\
$\Xi^{-} \rightarrow \Lambda$ & $\frac{2 m_{s}}{5 \sqrt{3}} M_{\Xi^{-}} \beta_{1}$ & 0.006 & 0.008 & -- \\
$\Xi^{-} \rightarrow \Sigma^{0}$ & $\frac{2 m_{s}}{15 \sqrt{3}} M_{\Xi^{-}}\left(21 \beta_{1}+16 \beta_{2}\right)$ & 0.125 & 0.04 & -- \\
\hline \hline
\end{tabular}

anstead of $g_{2} / f_{1}$, we list $\sqrt{\frac{3}{2}} g_{2}$. 


\section{REFERENCES}

[1] J. Ashman et al., Phys. Lett. B206, 364 (2988); Nucl. Phys. B328, 1 (1989).

[2] D. Adams et al., Phys. Lett. B329, 399 (1994); B. Adeva et al., Phys. Lett. B302, 533 (1993).

[3] P.L. Anthony et al., Phys. Rev. Lett. 71, 959 (1993).

[4] K. Abe et al., Phys. Rev. Lett., 74, 346 (1995).

[5] S.Y. Hsueh et al., Phys. Rev. D38, 2056 (1988).

[6] J. Dworkin et al. Phys. Rev. D41, 780 (1990).

[7] J.F. Donoghue and B.R. Holstein, Phys. Rev. D25, 206 (1982).

[8] A. Garcia and P. Kielanowski, "The beta decay of hyperons", Lecture Notes in Physics 222, Springer-Verlag, Heidelberg (1985).

[9] J.F. Donoghue, B.R. Holstein, and S.W. Klimt, Phys. Rev. D35, 934 (1987).

[10] M. Roos, Phys. Lett. B246, 179 (1990).

[11] C. Avenarius, Phys. Lett. B272, 71 (1991).

[12] B. Ehrnsperger and A. Schäfer, Phys. Lett. B348, 619 (1995).

[13] P.G. Ratcliffe, Phys. Lett. B365, 383 (1996).

[14] H.J. Lipkin, Phys. Lett. B214, 429 (1988); Phys. Lett. B230, 135 (1989).

[15] H.-C. Kim, M. V. Polyakov, A. Blotz, and K. Goeke, Nucl. Phys. A598, 379 (1996).

[16] A. Blotz, M. Praszałowicz, and K. Goeke, Phys. Lett. 317B, 195 (1993); Phys. Rev. D53, 485 (1996).

[17] D.I. Diakonov, V.Yu. Petrov, and P.V. Pobylitsa, Nucl. Phys. B306, 809 (1988).

[18] E. Witten, Nucl.Phys. B223, 433 (1983).

[19] G.S. Adkins, C.R. Nappi, and E. Witten, Nucl. Phys. B228, 552 (1983).

[20] J. Gasser and H. Leutwyler, Ann. Phys. 158, 142 (1984); Nucl. Phys. B250, 465 (1985).

[21] J. Wess and B. Zumino, Phys. Lett. 37B, 95 (1971).

[22] D.I. Diakonov and M.I. Eides, JETP Lett. 38, 433 (1983).

[23] I.J.R. Aitchison and C.M. Fraser, Phys. Rev. D31, 2605 (1985); D32, 2190 (1985).

[24] A. Dhar, R. Shankar, and S. Waida, Phys. Rev. D31, 3256 (1985).

[25] M. Praszałowicz, A. Blotz, and K. Goeke, Phys. Lett. B354, 415 (1995).

[26] H.-C. Kim, M.V. Polyakov, and K. Goeke, Phys. Rev. D53, R4715 (1996).

[27] C.V. Christov, A. Blotz, H.-C. Kim,P. Pobylitsa, T. Watabe, Th. Meissner, E. Ruiz Arriola and K. Goeke, Prog. Part. Nucl. Phys. 37, 91 (1996).

[28] D. Dyakonov and V. Petrov, Nucl.Phys. B272, 457 (1986); preprint LNPI-1153, published in: Hadron matter under extreme conditions, p.192, Kiew (1986).

[29] H.-C. Kim, A. Blotz, M. V. Polyakov, and K. Goeke, Phys. Rev. D53, 4013 (1996).

[30] M.V. Polyakov, Yad. Fiz. 51, 1110 (1990).

[31] H.-C. Kim, M.V. Polyakov, and K. Goeke, Phys. Rev. D55, 5698 (1997).

[32] A. Blotz, D. Diakonov, K. Goeke, N.W. Park, V. Petrov and P.V. Pobylitsa, Nucl. Phys. A555, 765 (1993).

[33] J.-M. Gaillard and G. Sauvage, Ann. Rev. Nucl. Part. Sci. 34, 351 (1984).

[34] J.J. De Swart, Rev. Mod. Phys. 35, 916 (1963).

[35] V. De Alfaro, S. Fubini, G. Furlan, and C. Rossetti, "Currents in hadron physics", NorthHolland Publishing Company, Amsterdam, (1973).

[36] M. Wakamatsu and H. Yoshiki, Nucl.Phys. A524, 561 (1991).

[37] A. Blotz, M.V. Polyakov, and K. Goeke, Phys. Lett. 302B, 151 (1993). 
[38] D. Diakonov, V. Petrov, and M. Polyakov, RUB-TPII-02/97, NORDITA-97/19N (hepph/9703373), to be published in Z. Phys. A (1997).

[39] V. Petrov, private communication (1997).

[40] J. Bijnens, H. Sonoda, and M.B. Wise, Phys. Lett. 140B, 421 (1984).

[41] M. Chemtob, Nuovo Cimento 89A, 381 (1985).

[42] S.J. Brodsky, J. Ellis, and M. Karliner, Phys. Lett. 206B, 309 (1988).

[43] M. Ademollo and R. Gatto, Phys. Rev. Lett. 13, 264 (1964).

[44] Particle Data Group, Phys. Rev. D54, part 1 (1996).

[45] M. Bourquin et al., Z. Phys. C12, 307 (1982).

[46] M. Bourquin et al., Z. Phys. C21, 1 (1983).

[47] N. Cabibbo, Phys. Rev. Lett. 10, 531 (1963).

[48] H.J. Lipkin, WIS-92/105/Dec-PH (hep-ph/9212316) (1992).

[49] F. Schlumpf, Phys. Rev. D51, 2262 (1995).

[50] N.W. Park and H. Weigel, Nucl. Phys. A541, 453 (1992).

[51] B.H. Kellett, Phys. Rev. 10, 2269 (1974). 